\title{
Severe kyphosis as a risk factor of umbilical trichophytosis in elderly people
}

\author{
Morikazu Onji1-3, Kaori Amano ${ }^{3}$, Katuyuki Matuki², Seiji Kondou², Tooru Hamada², \\ Shinya Furukawa ${ }^{1}$, and Teruki Miyake ${ }^{4}$ \\ ${ }^{1}$ Ehime University Graduate School of Medicine, Japan \\ ${ }^{2}$ Department of Internal Medicine, Kumakogen Town Hospital, Japan \\ ${ }^{3}$ Fujimine Clinic, Japan \\ ${ }^{4}$ Department of Gastroenterology and Metabology, Ehime University Graduate School of Medicine, Japan
}

\begin{abstract}
Objective: To determine whether severe kyphosis is one of the risk factors of umbilical trichophytosis in elderly people. Patients and Methods: Four cases with umbilical trichophytosis in the last 3 years and all 143 patients who visited to our clinic during from April 2018 to March 2019 were included in this study. The relationship between the condition of umbilical region and kyphosis was investigated. As regards the levels of senile kyphosis, simple visual classification of the kyphosis method was used (Level 0-4).

Results: All four cases with umbilical trichophytosis were elderly women aged $\geq 80$ years, had severe kyphosis, skin overlaps, filthy umbilical region, and lumps of dirt in the umbilical region and had nail tinea. Only two cases had umbilical trichophytosis among 143 patients who visited our clinic during the 1 year study period, with incidence is of $1.4 \% /$ year. About $65.7 \%, 60 \%$, and $37.1 \%$ of 35 patients with kyphosis with forward-bent posture had skin overlap, moist, and filthy umbilical regions, respectively. However, these symptoms were not found in patients without severe kyphosis.

Conclusion: Severe kyphosis with forward-bent posture could be one of the risk factors of umbilical trichophytosis in elderly people.
\end{abstract}

Key words: umbilical region, trichophytosis, kyphosis, elderly people

\section{Introduction}

Skin trichophytosis including the foot and hair, is very well known, even in developed and developing countries ${ }^{1}$. However, umbilical trichophytosis is classified as trichophytosis corporis, with incidence reported to be $0.68 \%$ in all trichophytosis ${ }^{2}$. Consequently, umbilical trichophytosis would be extremely rare. Trichophytosis more commonly occurs in elderly people than that in younger patients ${ }^{3}$. $\mathrm{Ku}-$

Received: December 8, 2019

Accepted: March 23, 2020

Correspondence: Morikazu Onji, MD, Ehime University Graduate School of Medicine, 1303-2 Shizukawa, Toon, Ehime 791-0203, Japan

E-mail: onji05on@gmail.com

This is an open-access article distributed under the terms of the Creative Commons Attribution Non-Commercial No Derivatives (by-nc-nd) License $<$ http://creativecommons.org/ licenses/by-nc-nd/4.0/>. makogen town is located in the Southwest Part of Shikoku Mountains of Japan. In this area, the population density is progressively decreasing with an increase in aging population. About $47 \%$ of the population of this area are over 65 years old ${ }^{4}$.

Recently, two patients with umbilical trichophytosis lesions were presented to Fujimine clinic, Kumakogen town, Ehime, Japan, in the last year, and four with umbilical trichophytosis were found during in the last 3 years. All four patients with umbilical trichophytosis were aged $\geq 80$ years, with skin overlap, moist, and filthy umbilical regions due to severe kyphosis and trichophytosis in the foot or nail. Reports showed that farmers in the farm village suffered from severe kyphosis ${ }^{5}$. Thereupon, the incidence of kyphosis, skin overlap, moist, and filthy umbilical regions was examined in patients who visited Fujimine clinic in the last year (from April 2018 to March 2019) in present study. Therefore, this study primarily aimed to assess whether severe kyphosis is a risk factor for umbilical trichophytosis. 
Table 1 Summary of clinical manifestations of 4 cases with umbilical trichophytosis

\begin{tabular}{|c|c|c|c|c|}
\hline Case & 1 & 2 & 3 & 4 \\
\hline Sex & Female & Female & Female & Female \\
\hline Age & 80 & 86 & 84 & 92 \\
\hline Season of onset & April & June & May & Feb \\
\hline Body weight (kg), BMI & $31,18.3$ & $53,23.8$ & 45,22 & 27,17 \\
\hline Nutrition state & Slightly poor & Good & Good & Poor \\
\hline Serum Alb. Level (g/dL) & 3.8 & 3.9 & 3.8 & 3.6 \\
\hline WBC & 4,970 & 5,890 & 4,810 & 3,900 \\
\hline Lymphocyte & $1,322(26.6 \%)$ & $1,785(32 \%)$ & $2,208(46 \%)$ & $580(16 \%)$ \\
\hline Complication of diabetes & $(-)$ & $(-)$ & $(-)$ & $(-)$ \\
\hline Kyphosis (Level) & Severe (4) & Severe (4) & Severe (3) & Severe (4) \\
\hline Forward-bent posture & $\geq 90^{\circ}$ & $\geq 90^{\circ}$ & $\geq 45^{\circ}$ & $\geq 90^{\circ}$ \\
\hline Condition of umbilical region & $\begin{array}{l}\text { Skin overlap } \\
\text { moist, filthy }\end{array}$ & $\begin{array}{l}\text { Skin overlap } \\
\text { moist, filthy }\end{array}$ & $\begin{array}{l}\text { Skin overlap } \\
\text { moist, filthy }\end{array}$ & $\begin{array}{l}\text { Skin overlap } \\
\text { moist, filthy }\end{array}$ \\
\hline Trichophytosis in other organs & $\begin{array}{c}\text { Foot, nail, hands } \\
\text { pubic region }\end{array}$ & Foot, nail & Foot, nail & Foot, nail \\
\hline Lesions range & Entire abdomen & $\begin{array}{c}\text { Localized range } \\
\text { of radius } 5 \mathrm{~cm}\end{array}$ & $\begin{array}{c}\text { Localized range } \\
\text { of radius } 5 \mathrm{~cm}\end{array}$ & $\begin{array}{c}\text { Localized range } \\
\text { of radius } 5 \mathrm{~cm}\end{array}$ \\
\hline Cleaning of umbilical region & $\begin{array}{c}\text { Day service } \\
\text { bathing } 1 / \text { week }\end{array}$ & $\begin{array}{c}\text { Day service } \\
\text { bathing } 1 / \text { week }\end{array}$ & $\begin{array}{c}\text { Wash herself } \\
\text { every day }\end{array}$ & $\begin{array}{c}\text { No bathing for } \\
1 \text { month }\end{array}$ \\
\hline Treatment & Lulicozole & Ketoconazole & Ketoconale & Ketoconale \\
\hline Relapse & 4 times & 2 times & 2 times & No for 6 months \\
\hline Prognosis & Died at $84 \mathrm{y} / \mathrm{o}$ & Live & Live & Died at $92 \mathrm{y} / \mathrm{o}$ \\
\hline Living with & Husband & Husband & Alone & Son \\
\hline Visiting nursing \& care management & $(-)$ & $(-)$ & $(-)$ & $(-)$ \\
\hline
\end{tabular}

\section{Patients and Methods}

Patients with umbilical trichophytosis in Fujimine clinic were examined for the last 3 years (Table 1). Trichophytosis was diagnosed only based on clinical features as described: skin lesion, existence of nail and/or foot trichophytosis, and effect with antifungals. No direct evidence was found for the diagnosis of trichophytosis, such as the direct microscopic examination of clinical samples, histopathology, culture, and serology ${ }^{6}$.

Patients who visited the Fujimine Clinic in the last year (from April 2018 to March 2019) were examined, and the incidence of kyphosis (Level 2-4 shown in Figure 1), skin overlap, moist and filthy umbilical regions, nail and/or foot trichophytosis, diabetes mellitus, nutritional state, and body mass index (BMI) were also investigated.

A total of 143 patients who visited the clinic from April 2018 to March 2019 were included. As regards the levels of senile kyphosis, visual classification of kyphosis method was used, because it is very convenient without training examiners using a specialized equipments such as X-rays. Our visual classification of kyphosis was performed by Dr. Onji and Mrs. Amano (nurse), independently observing patient's natural walking ability in the clinic. The levels of kyphosis were graded as no kyphosis (Level 0), the slight kyphosis
(Level 1) without forward-bent posture, moderate kyphosis (Level 2) with forward-bent posture (approximately 10-40 of the lumbar spine), severe kyphosis (Level 3) with forwardbent posture ( $\geq$ approximately $45^{\circ}$ of the lumbar spine), and very severe kyphosis (Level 4) with forward-bent posture $(\geq$ approximately $90^{\circ}$ of lumbar spine) (Figure 1). The forwardbent posture in Levels 2, 3, and 4 were measured by eyes, based on the angle between the line connecting the part of 7 th cervical and 5 th lumbar spinous processes, and the vertical line through 5 th lumbar process. Although our method is one of the visual classifications of kyphosis in the Kurabuchi study described in 20197), our method was independent of this report.

The incidences of skin overlap, moist and filthy umbilical region, nail and/or foot trichophytosis were compared between 15 patients without kyphosis (Level 0 and 1) and 35 with kyphosis (Level 2, 3, and 4) (Figure 2). As regards the patients who visited Fujimine clinic, the incidence of kyphosis was investigated from April 2018 to March 2019 (Figure 3).

For statistical processing, a $\chi^{2}$ test was used, and a significant difference test was conducted at a significance level of less than $(\leq) 5 \%$.

The present study was approved by Kumakogen Town Hospital Ethics Committee (approval number I-1-2018). 


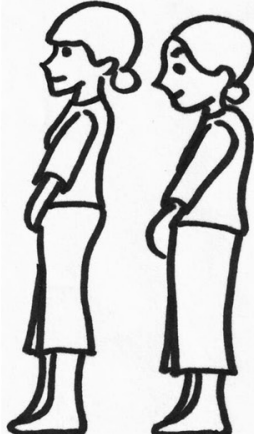

Levels

Kyphosis of Cervical \& Thoracic Spine Kyphosis with Forward-bent posture

0

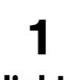

slight

none

slight
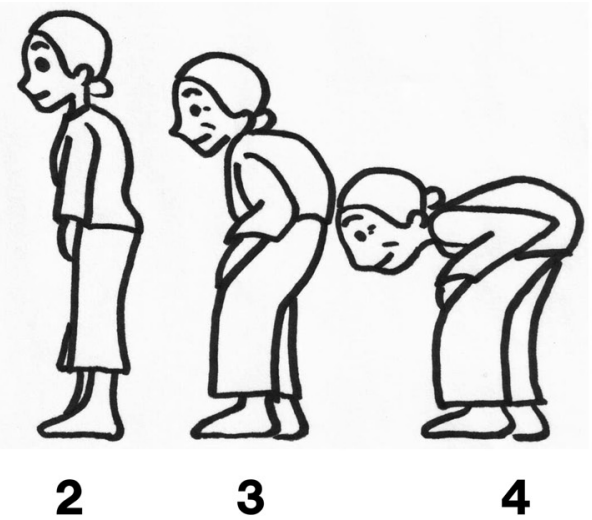

moderate

3

4

present

severe

present

Figure 1 Visual classification of kyphosis levels.

Our visual classification of kyphosis was performed by observing patient's normal walking ability in the clinic. The kyphosis levels are no kyphosis (Level 0), very slight kyphosis without forward-bent posture (Level 1), moderate kyphosis with forward-bent posture (approximately $10^{\circ}-40^{\circ}$ of the lumbar spine) (Level 2), severe kyphosis with forward-bent posture (more than $(\geq)$ approximately $45^{\circ}$ of the lumbar spine) (Level 3), and very severe kyphosis with forward-bent posture (more than $\left(\geq\right.$ ) approximately $90^{\circ}$ of the lumbar spine) (Level 4). The forward-bent posture in Levels 2, 3, and 4 were measured by eyes, based on the angle between the line connecting the part of 7 th cervical and 5 th lumbar spinous processes, and the vertical line through 5 th lumbar process. The forward-bent posture in Levels 2, 3, and 4 were measured by eyes, based on the angle between the line connecting the part of 7 th cervical and 5 th lumbar spinous processes, and the vertical line through 5 th lumbar process.

\section{Results}

Four cases of umbilical trichophytosis were identified for the last 3 years in Fujimine Clinic, although umbilical trichophytosis rarely occurs. All data of 4 cases are shown on Table 1. Among the four cases of umbilical trichophytosis, three had the skin lesions of trichophytosis localized in a radius of about approximately $5 \mathrm{~cm}$ of the umbilical region, although the skin lesion of umbilical trichophytosis usually occurs as part of trichophytosis in the abdomen and/or perineum. All cases were the elderly women aged $\geq 80$ years old, had severe kyphosis (Level 3-4), skin overlaps, filthy umbilical region and lumps of dirt in the umbilical region and nail and/or foot trichophytosis. Umbilical trichophytosis lesions were improved with anti-fungal administration for 4 weeks, but the recurrence was observed in 3 of 4 cases. In 2 of 4 cases, BMI was within normal limits. Two of 4 cases had poor nutritional status, but their blood albumin levels were within normal limits. No case had diabetes mellitus. No case was gotten underwent the regular medical cares at home by a professional care manger. The keyperson for their cases at home were their husbands or son.

The number of patients visited the clinic was 143 for the 1 year (April 2018 to March 2019). Factors related with kyphosis, which should be considered as a risk factor of umbilical trichophytosis, were compared between 35 patients with moderate-severe kyphosis (Levels 2-4), and 15 patients without kyphosis (Level 0-1), (Figure 2). All four cases with umbilical trichophytosis had very severe kyphosis (Level 3, $4)$. The difference in ages among four cases of umbilical trichophytosis $(85 \pm 5.0, \mathrm{n}=4)$ and 35 patients with kyphosis $(85.4 \pm 5.4, \mathrm{n}=35)$ was not significant $(P=0.78)$. Age of 35 patients $(85.4 \pm 5.4)$ with kyphosis (Level $2-4)$ is significantly higher than those 15 patients without severe kyphosis (Level $0-1 \quad(81.3 \pm 4.1)(P<0.01)$ (Figure 2). The difference in the incidence of foot/nail trichophytosis between 15 patients without kyphosis and 35 patients with kyphosis was not significant ( $P=0.58$ ) (Figure 2 ). These data could suggest that the kyphosis is a more important risk factor of umbilical trichophytosis than age. 

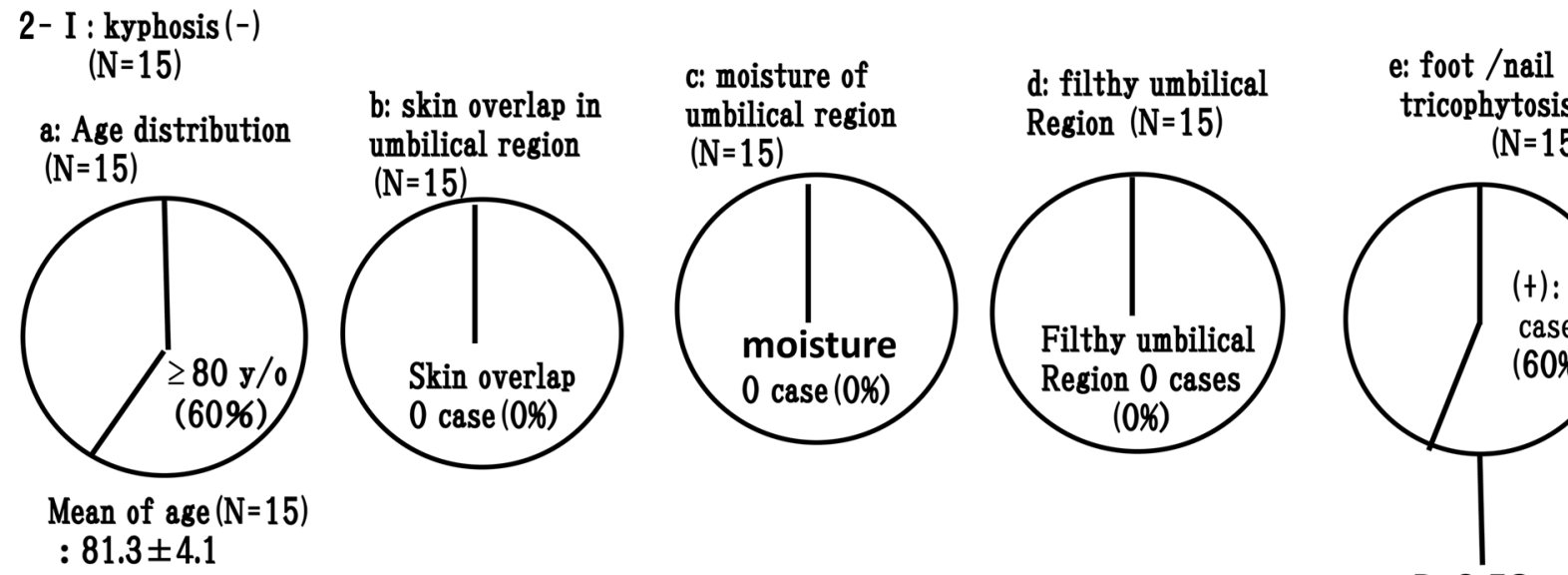
tricophytosis umbilical region $(\mathrm{N}=15)$
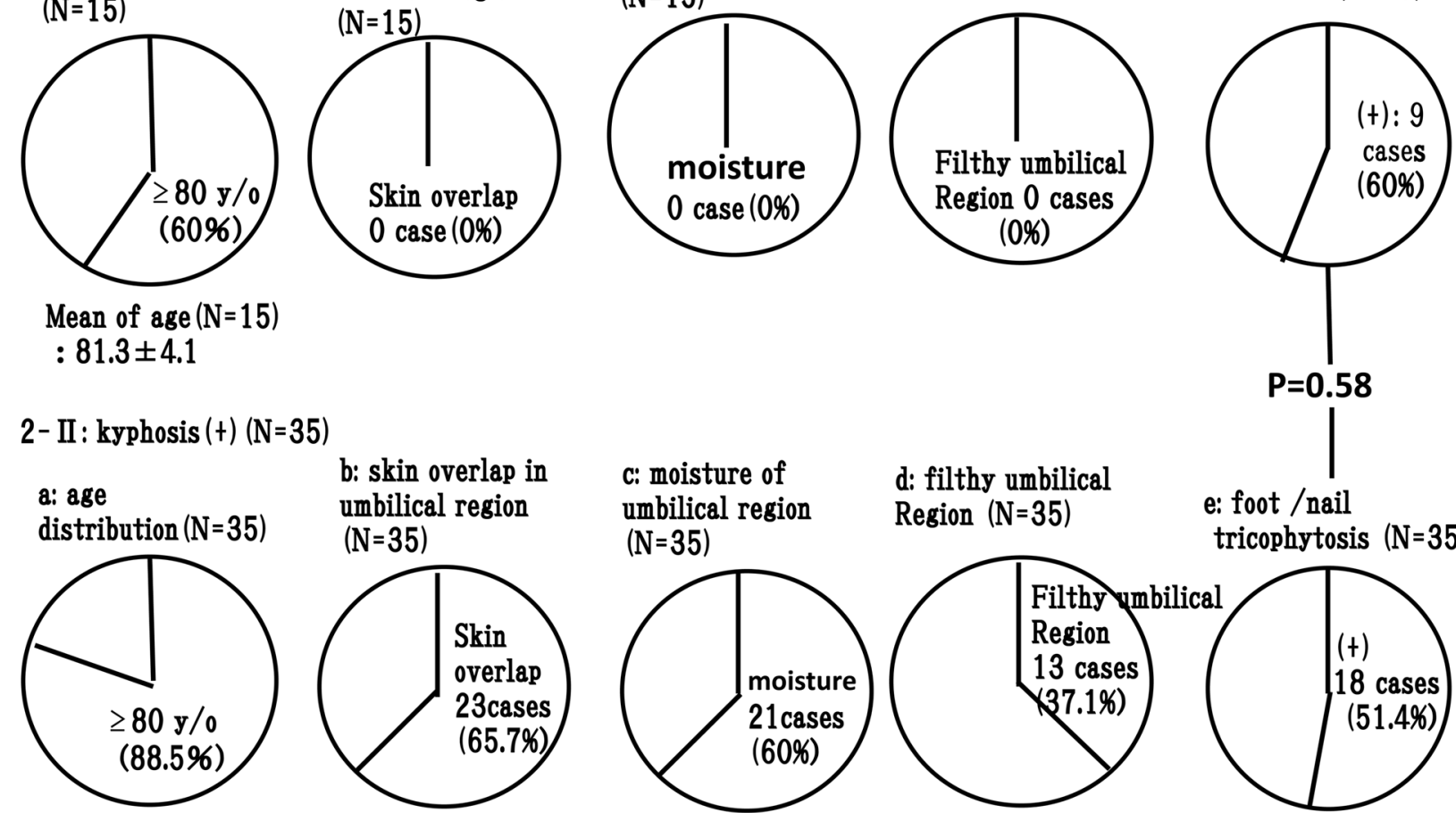

b: skin overlap in umbilical region $(\mathrm{N}=35)$
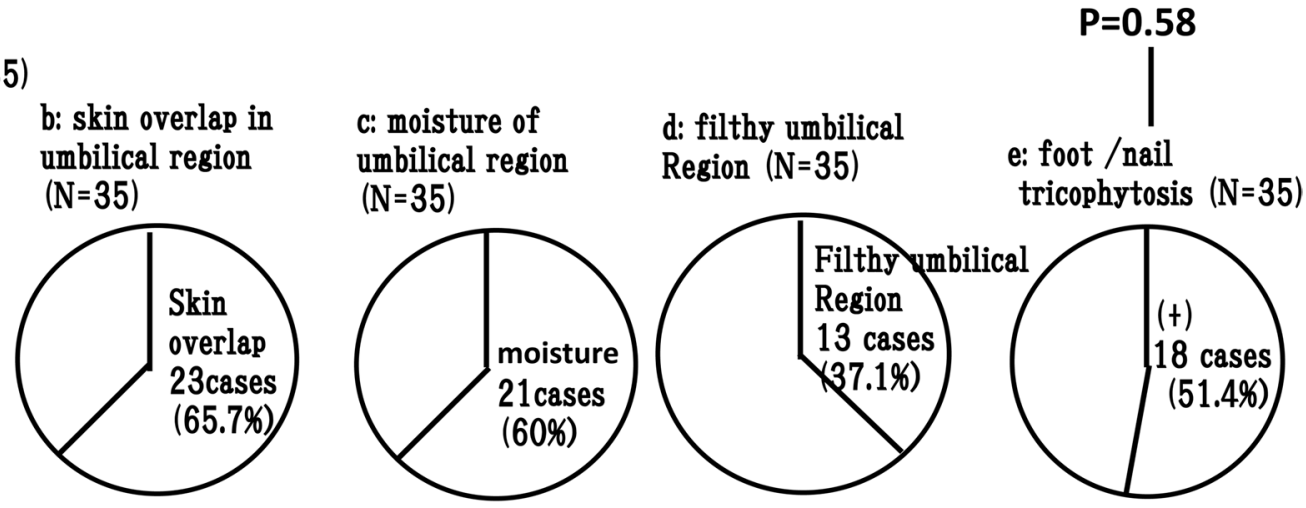

: foot / nail

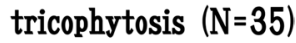

Mean of age $(\mathrm{N}=35)$ : $85.4 \pm 5.4$

Figure 2 Difference of factors related to kyphosis between with and without kyphosis.

Factors related with kyphosis (Level 3-4), which should be considered as a risk factor of umbilical trichophytosis were compared between 35 patients with and 15 without kyphosis, who visited Fujimine clinic during the 1-year study period, from April 2018 to March 2019. Age of 35 patients (age:85.4 \pm 5.4 ) with kyphosis is significantly higher than 15 patients without kyphosis (Level 0-1), (age:81.3 \pm 4.1$)(P<0.01)$. Incidences of skin overlap in the umbilical region, moist, filthy umbilical regions in patients with kyphosis, i.e., $65.7 \%, 60 \%$ and $37.1 \%$, respectively, are higher than those without, i.e., $0 \%$, $0 \%$, and $0 \%$, respectively. In 35 patients with kyphosis, nail and/or foot trichophytosis was observed in 18 cases $(51.4 \%)$, which is not significantly higher than 15 cases without kyphosis $(60 \%)$.

Incidences of skin overlap in the umbilical region, moist, and filthy umbilical regions in patients with kyphosis (Level $2-4$ ) were higher, i.e., $65.7 \%, 60 \%$, and $37.1 \%$, respectively, than those without it (Level $0-1$ ), i.e., $0 \%, 0 \%$, and $0 \%$, respectively. In 35 patients with kyphosis (Level 2-4), nail and/or foot trichophytosis was observed in 18 cases (51.4\%). This incidence is not significantly higher than 15 cases without severe kyphosis (Level 0-1) (60\%). Skin overlap occurred in 5 of 11 cases $(45.5 \%)$ in patients with moderate kyphosis and, 18 of 24 cases (75\%) with severe kyphosis (Level 3-4). Filthy umbilical region occurred in 3 of 11 cases $(27.3 \%)$ in patients with moderate kyphosis (Level 2) and, 10 of 24 cases $(41.7 \%$ ) with severe kyphosis (Level 3-4). However, no significant difference was observed between the two groups. In 35 patients with moderate-severe kyphosis (Level $2-4)$, only 3 patients $(8.6 \%$ ) had diabetes mellitus and, $4(11.4 \%)$ had poor nutritional status. These patients showed the mean BMI was of $22.4 \pm 3.5$. Four cases $(11.4 \%)$ were obese with $\geq 25 \mathrm{BMI}$, and same number of patients $(11.4 \%)$ had lean body stature with $<18.5$ BMI.

Relationship between age distribution of patients and existence of kyphosis was compared (Figure 3-1). A total of 76 patients aged $\geq 80$ years old were $76(53 \%)$ and only 19 aged $<65$ years old $(13 \%)$. Visual kyphosis levels in the spinal column showed the number of patients in each Levels (0.1.2.3.4) were 33 (23.1\%), 21 (14.7\%), 49 (34.2\%), 34 (23.8\%), and 6 (4.2\%), respectively (Figure $3-2$ ). The number of patients without kyphosis, with moderate kyphosis (Level 2), and with severe kyphosis (Level 3-4) was 54 (34\%), 49 (34\%), and $40(28 \%)$, respectively. Kyphosis levels were higher in elderly people as shown in Figures 3-3, 4, and 5. 
3-1 : age distribution ( $N=143)$

Mean of age $(\mathrm{N}=143): 76.8 \pm 12.3$

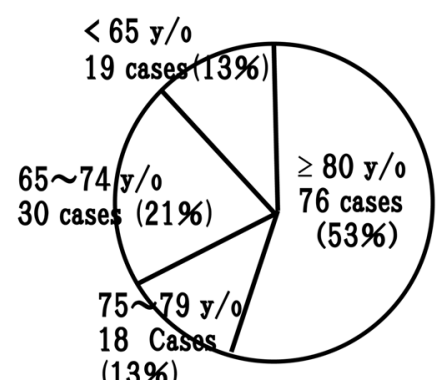

\section{3-2 : kyphosis $(\mathrm{N}=143)$}

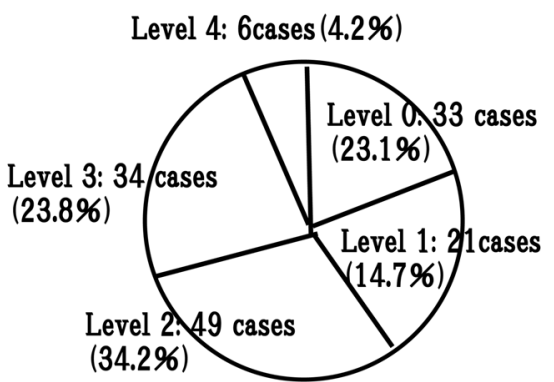

\section{3-3: kyphosis (Level: 0-1)} $(\mathrm{N}=54)$

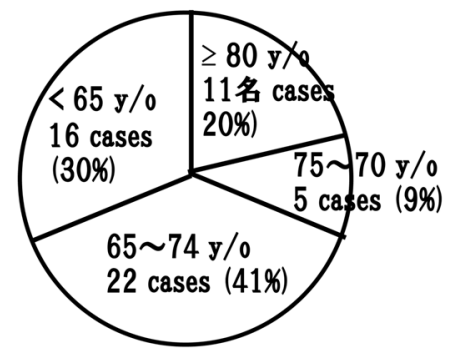

3-4 : (Level: 2) $(\mathrm{N}=49)$

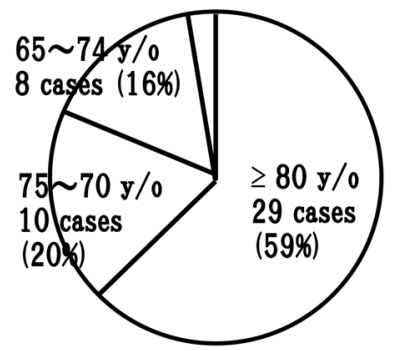

3-5 : (Level: 3-4) $(\mathrm{N}=40)$

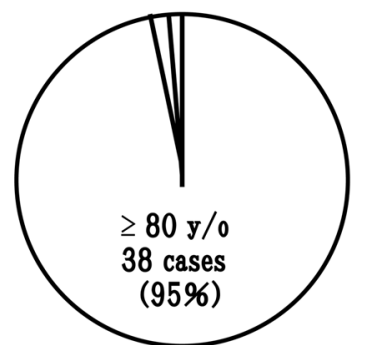

Figure 3 Relationship between age distribution of patients and existence of kyphosis.

As the number of patients who visited the clinic was 143 during the 1-year study period (from April 2018 to March 2019). Relationship between age distribution of patients and existence of kyphosis was compared (Figure 3-1). Patients aged $<65$ years were only $19(13 \%)$. Patients aged $\geq 80$ years were $76(53 \%)$. Visual kyphosis levels in the spinal column showed number of patients in each Level (0.1.2.3.4) were $33(23.1 \%), 21(14.7 \%), 49(34.2 \%)$, $34(23.8 \%)$, and $6(4.2 \%)$, respectively (Figure 3-2). The number of patients without kyphosis and with kyphosis without forward-bent posture, with moderate kyphosis (Level 2), and with severe kyphosis (Level 3-4) were 54 (34\%), 49 (34\%), and 40 (28\%), respectively. Kyphosis levels were higher in elderly people (Figure 3-3, 4, and 5).

\section{Discussion}

Aging population is increasing internationally, particularly in many countries, such as Japan, Korea, and China. The growth of aging population in Japan is going the fastest in the worldwide ${ }^{8)}$. Japan's experiences about severe aging society should provide for the helpful measures for other countries. Kumakogen town is located in the Southwest region of Shikoku Mountains in Shikoku Island, Japan. In this area, depopulation is rapidly progressing, and aging is most advanced with $\geq 47 \%$ of population aged $\geq 65$ years. This area had the highest aging rate in Ehime Prefecture, which had the 8th aging rate $(30.6 \%)$ in Japan in $2015^{4}$. Kumakogen town can be considered as an advanced district with an aging society. Therefore, many people had kyphosis, especially in the rural district and/or farming area ${ }^{5)}$.

Several risk factors for trichophytosis, and nail and/or foot trichophytosis were reported to be high in places with high average temperature, male, elderly people, family history, vascular diseases, diabetes mellitus, obesity, hyper- cholesterolemia, wound, bone and joint diseases, sports, liver diseases, kidney diseases, acquired immunodeficiency syndrome, malignant diseases, use of immunosuppressant, steroids, and anti-cancer drugs, etc ${ }^{9}{ }^{10)}$. The risk factor of umbilical trichophytosis has not been reported yet, because this is a rare disease entity. Moreover, severe kyphosis would be included as a risk factor of elderly people. Trichophytosis is well known, especially in elderly people even in developed and developing countries. However, trichophytosis corporis rarely occurs, accounting for only $0.68 \%$ of all trichophytosis ${ }^{2}$. However, our survey on the incidence of umbilical trichophytosis is $1.4 \%$ /year, which should be very high. Severe kyphosis (Level 3-4), which induces skin overlap as shown in Figure 2, can be considered as one of the causes of high of incidence of umbilical trichophytosis. Skin overlap should induce moist and filthy conditions in the umbilical region, which promotes the occurrence of infection and growth of trichophyton according to our hypothesis. Prophylaxis of infection and inhibition of trichophytosis growth become difficult due to severe kyphosis. In the 
present study, localization of umbilical trichophytosis lesion in 3 of 4 cases, should be considered as a special feature. This characteristic finding, localization of umbilical lesion, would occur due to senile kyphosis.

Other main factors that induce the occurrence of filthy umbilical region are insufficient washing/cleaning of the umbilical region. This usually occurs due to skin overlap induced by severe kyphosis with forward-bent posture. Further, kyphosis itself would prevent washing/cleansing of the umbilical region. As the skin overlap was exacerbated due to severe kyphosis, washing the umbilical region becomes more difficult, because it requires the patient to bend forward to wash the umbilical region. The existence of old people providing care for old people is present Japan, and care at home by non-professional persons (husbands or son) (Table 1).

This study had several limitations, i.e., small number of patients, and different methods used to diagnose trichophytosis and kyphosis. Umbilical trichophytosis was diagnosed based on clinical features including skin lesions only. Although the detection rate of trichophyton is very low, no direct evidence, such as direct microscopic examination of clinical samples, histopathology, culture, and serology, was performed in clinics with few facilities. As regards the senile kyphosis levels, the visual classification of kyphosis method was used, because of its convenience and without the need of trained examiners using specialized equipments such as X-rays. The kyphosis levels are no kyphosis, very slight kyphosis without forward-bent posture, moderate kyphosis with forward-bent posture (approximately $10-40^{\circ}$ of lumbar spine), severe kyphosis with forward-bent posture ( $\geq$ approximately $45^{\circ}$ of lumbar spine), and very severe kyphosis with forward-bent posture ( $\geq$ approximately $90^{\circ}$ of lumbar spine) (Figure 1). Our method is one of the visual classifications for kyphosis in Kurabuchi's study conducted in 20197). However, our method was independent of Kurabuchi's study. The estimation of kyphosis levels should be more detailed in severe kyphosis in this study, because we included many patients with severe kyphosis.

In conclusion, severe kyphosis with forward-bent posture could be one of the risk factors of umbilical trichophytosis in elderly people. Therefore, the umbilical region of patients with severe senile kyphosis should be more carefully washed by a professional person.

\section{Acknowledgments}

The authors are grateful to Mrs. Yasuko Nishimura (Fujimine clinic, Kou 415-2, Tsuyumine, Kumakogen town, Ehime, Japan) for her helpful assistance in getting the required information from patients, and Mr. Jyunpei Takimoto and Mrs. Iwane Oono (Department of Rehabilitation, $\mathrm{Ku}-$ makogen Town Hospital, Kumakogen town, Ehime, Japan) for their drawing in Figure 1 and for providing orthopedic information.

\section{References}

1. Watanabe S, Harada T, Hiruma M, et al. Japan Foot Week Group. Epidemiological survey of foot diseases in Japan: results of 30,000 foot checks by dermatologists. J Dermatol 2010; 37: 397-406. [Medline] [CrossRef]

2. Sei Y. [2011 Epidemiological Survey of Dermatomycoses in Japan]. Med Mycol J 2015; 56: J129-J135 (in Japanese). [Medline] [CrossRef]

3. Harada T. [Tinea unguium]. Med Mycol J 2011; 52: 77-95 (in Japanese). [Medline] [CrossRef]

4. https://www.pref.ehime.jp/h20400/koureisyajinkou.html.

5. Ooniwa H. Research on survey of lumbago in one forestry mountain village. Nihon Noson Igakkai Zasshi (JJRM) 1977; 26: 152-159 (in Japanese).

6. https://www.aafp.org/afp/2002/0515/p2095.html.

7. Sugai K, Michikawa T, Takebayashi T, et al. Association between visual classification of kyphosis and future ADL decline in community-dwelling elderly people: the Kurabuchi study. Arch Osteoporos 2018; 14: 3. [Medline] [CrossRef]

8. https://www.tyojyu.or.jp/net/kenkou-tyoju/tyojyu-shakai/sekaiichi.html.

9. Qadim HH, Golforoushan F, Azimi H, et al. Factors leading to dermatophytosis. Ann Parasitol 2013; 59: 99-102. [Medline]

10. Ogasawara Y. [Prevalence and patient's consciousness of tinea pedis and onychomycosis]. Nippon Ishinkin Gakkai Zasshi 2003; 44: 253-260 (in Japanese). [Medline] [CrossRef] 